\title{
SUPERCRITICAL BEHAVIOR OF MAGNETIC AND LIQUID MODEL SYSTEMS
}

\author{
A.N. Galdina \\ Oles Honchar Dnipro National University, Dnipro, Ukraine \\ e-mail: alexandragaldina@gmail.com
}

The supercritical transitions are widely occurring. They include the supercritical transitions in the liquid-vapor system, ferromagnetic transitions, transitions in polymers, many transitions in liquid crystals, and some structural transitions. In the paper it is emphasized that the nature of the critical and supercritical transitions is the same - these are continuous fluctuation transitions. Above the critical temperature the system passes through a region of lowered stability, which leads to increase of fluctuations of energy and external parameters of the system. From the point of view of thermodynamic stability this indicates the existence of a continuous supercritical transition between supercritical mesophases. Knowing the basic stability characteristics of a system, we derive the equation of these mesophase transitions. Depending on a thermal equation type, we can get one or several such equations, which may not coincide. This approves the fact that a supercritical transition occurs in a certain interval of thermodynamic forces.

In the paper the relations between the critical exponents of thermodynamic parameters of the system are obtained and the conditions of continuous conjugation of the lowered stability line to subcritical coexistence line are investigated. The results are applied to the Curie-Weiss and van der Waals models: we obtain the quasi-spinodal equation for these systems and analyze the critical and supercritical behavior of the stability characteristics.

Keywords: critical exponents, coexistence curve, critical slope, thermodynamic stability, critical state, supercritical phase transitions.

Received 12.11.2019; Received in revised form 15.12.2019; Accepted 23.12.2019

\section{Introduction}

Above the critical point, the coefficients of stability and the determinants of stability are known to pass through continuous minima, the depth of which decreases with a distance from the critical point. It obviously means that in the supercritical region the fluctuations do not decay and decrease apart the critical point. This fact testifies that the supercritical region becomes microheterogeneous within an interval of thermodynamic forces, which indicates that properties of the subcritical phases are retained above the critical point. Analyzing the experimental data from the point of view of thermodynamic stability leads to the conclusion [1] that there is a continuous supercritical transition between the supercritical quasi-phases. It is a passing of the system through the region of the lowered stability that corresponds to the growth of fluctuations. The continuous character of the transition is due to the fact that it occurs outside the region of instability.

The curves of the lowered stability may not coincide for different coefficients of stability. This is a confirmation of the fact that the supercritical transition takes place within a certain range of thermodynamic forces. Conditionally, transition temperature is a temperature corresponding to the minimum of the determinant of stability, because $D$ describes best the stability of a system.

This study flows organically from the paper [2]. We examine the features of supercritical and critical transitions in liquid and magnetic systems and apply the findings to so-called "classical models" of the phase transitions, namely, to the van der Waals and CurieWeiss models. Such choice is dictated by the fact, that their thermodynamic properties are known in details to compare them with our results. Besides, the "mean field" models (including the van der Waals model) are still widely used for comparative qualitative analysis and modeling the behavior of real physical systems, such as alloys and melts [3] and, what is more, the nuclear matter [4-6]. 


\section{The quasi-spinodal equation and relationship between the critical exponents}

To fix the idea, let us consider a one-component $P-V-T$ system, the number of particles is constant $N=$ const . In the paper [2] from the quasi-thermodynamic theory of fluctuations we obtained

$$
\left(-\frac{\partial^{2} P}{\partial V^{2}}\right)_{T}=0
$$

for the lowered stability curve, and that from the thermodynamic stability theory follows $d D=0$.

In general, we consider the determinant of stability as a function of entropy and volume. So, we have

$$
d D=\left(\frac{\partial D}{\partial S}\right)_{V} d S+\left(\frac{\partial D}{\partial V}\right)_{S} d V=0 \quad \text { or }\left(\frac{\partial D}{\partial S}\right)_{V}=0, \quad\left(\frac{\partial D}{\partial V}\right)_{S}=0
$$

But, in practice, when we are working out the problem based on the thermal state equation, which includes the observables, we often obtain $D$ in terms of volume and temperature. Thus, we have to rewrite (2) in terms of $V, T$ :

$$
\begin{aligned}
& \left(\frac{\partial D}{\partial S}\right)_{V}=\frac{\partial(D, V)}{\partial(T, V)} \frac{\partial(T, V)}{\partial(S, V)}=\left(\frac{\partial T}{\partial S}\right)_{V}\left(\frac{\partial D}{\partial T}\right)_{V}=0 \\
& \left(\frac{\partial D}{\partial V}\right)_{S}=\frac{\partial(D, S)}{\partial(V, T)} \frac{\partial(V, T)}{\partial(V, S)}=\left(\frac{\partial D}{\partial V}\right)_{T}-\left(\frac{\partial T}{\partial S}\right)_{V}\left(\frac{\partial D}{\partial T}\right)_{V}\left(\frac{\partial P}{\partial T}\right)_{V}=0 .
\end{aligned}
$$

As $(\partial T / \partial S)_{V} \geq 0$ according to the requirements of thermodynamic stability, $(\partial T / \partial S)_{V}=0$ at the critical point only, and $(\partial P / \partial T)_{V}=$ const $\neq 0$, then we have a set of equations:

$$
\left(\frac{\partial D}{\partial T}\right)_{V}=0, \quad\left(\frac{\partial D}{\partial V}\right)_{T}=0
$$

To calculate the determinant of stability we can use the definition of the determinant of stability [7] and the derived relations [8-9]:

$$
\begin{aligned}
& D=\left(\frac{\partial T}{\partial S}\right)_{V}\left(-\frac{\partial P}{\partial V}\right)_{T}=\left(\frac{\partial T}{\partial S}\right)_{P}\left(-\frac{\partial P}{\partial V}\right)_{S}=-\left(\frac{\partial T}{\partial V}\right)_{P}\left(\frac{\partial T}{\partial V}\right)_{S}= \\
& =\left(\frac{\partial T}{\partial S}\right)_{V}\left(-\frac{\partial P}{\partial V}\right)_{S}-\left(\frac{\partial T}{\partial V}\right)_{S}^{2} .
\end{aligned}
$$

For simplicity we take the first term of (4): $D=(\partial T / \partial S)_{V}(-\partial P / \partial V)_{T}=T(-\partial P / \partial V)_{T} / C_{V}$. We find its derivatives as

$$
\left(\frac{\partial D}{\partial T}\right)_{V}=\frac{\partial}{\partial T}\left[\left(\frac{\partial T}{\partial S}\right)_{V}\left(-\frac{\partial P}{\partial V}\right)_{T}\right]=0, \quad\left(\frac{\partial D}{\partial V}\right)_{T}=\frac{\partial}{\partial V}\left[\left(\frac{\partial T}{\partial S}\right)_{V}\left(-\frac{\partial P}{\partial V}\right)_{T}\right]=0 .
$$

In general case, considering $C_{V}=C_{V}(T, V)$, we get 


$$
\begin{aligned}
& \left(\frac{\partial D}{\partial T}\right)_{V}=\frac{1}{C_{V}}\left[\left(-\frac{\partial P}{\partial V}\right)_{T}\left(1-\frac{T}{C_{V}}\left(\frac{\partial C_{V}}{\partial T}\right)_{V}\right)+T\left(-\frac{\partial^{2} P}{\partial T \partial V}\right)\right]=0, \\
& \left(\frac{\partial D}{\partial V}\right)_{T}=\frac{\partial}{\partial V}\left[\left(\frac{\partial T}{\partial S}\right)_{V}\left(-\frac{\partial P}{\partial V}\right)_{T}\right]=\frac{T}{C_{V}}\left[-\frac{1}{C_{V}}\left(\frac{\partial C_{V}}{\partial V}\right)_{T}\left(-\frac{\partial P}{\partial V}\right)_{T}+\left(-\frac{\partial^{2} P}{\partial V^{2}}\right)_{T}\right]=0 .
\end{aligned}
$$

For systems, which thermal equation is linear in temperature, heat capacity is $C_{V}=C_{V}^{\text {id }}$ where $C_{V}^{\text {id }}$ is the heat capacity of an ideal gas. Therefore, for such a system we obtain $(\partial D / \partial T)_{V} \equiv 0$ and $(\partial D / \partial V)_{T}=\left(-\partial^{2} P / \partial V^{2}\right)_{T}$, which is the result of quasithermodynamic fluctuation consideration of supercritical transitions [2]. And the curve of lowered stability of the $D$ coincides with that for isodynamic mechanical coefficient of stability $(-\partial P / \partial V)_{T}$. For a generalized system with $C_{V}=C_{V}(T, V)$ there is a shift in the equations for $D(5)$ and thus we have to consider the lowered stability just for $D$ to obtain the equation of the quasispinodal.

The same can be derived for the rest isodynamic and adiabatic characteristics of stability and another definition of $D(4)$.

Let us consider the subcritical region. For the linear phase thansition, i.e. the first kind phase transitions, which equilibrium curve is the line, the eqiation of the coexistense line is

$$
\frac{d P}{d T}=\frac{\Delta S}{\Delta V}=\text { const } .
$$

If we evaluate the numerator and the denominator, we can write

$$
\frac{d P}{d T}=\frac{\left(\frac{\partial S}{\partial T}\right)_{P} \Delta T+\left(\frac{\partial S}{\partial P}\right)_{T} \Delta P}{\left(\frac{\partial V}{\partial T}\right)_{P} \Delta T+\left(\frac{\partial V}{\partial P}\right)_{T} \Delta P}=\text { const },
$$

that is, the numerator and the denominator have to be of the same pattern of change:

$$
\left(\frac{\partial S}{\partial T}\right)_{P} \sim\left(\frac{\partial V}{\partial T}\right)_{P},\left(\frac{\partial S}{\partial P}\right)_{T}=\left(-\frac{\partial V}{\partial T}\right)_{P} \sim\left(\frac{\partial V}{\partial P}\right)_{T}
$$

Let us approach the critical point along the equilibrium line in temperature and introduce for its small neighborhood the critical exponents according to their common definition [10]

$$
C_{P}=T\left(\frac{\partial S}{\partial T}\right)_{P} \sim(-\tau)^{-\alpha^{\prime}} ; \quad K_{T}=\frac{1}{V}\left(-\frac{\partial V}{\partial P}\right)_{T} \sim(-\tau)^{-\gamma^{\prime}} ; \quad B_{p}=\frac{1}{V}\left(\frac{\partial V}{\partial T}\right)_{P} \sim(-\tau)^{\mu^{\prime}},
$$

where $C_{P}, K_{T}, B_{p}$ are isobaric heat capacity, compressibility coefficient and thermal expansivity, correspondingly; $\tau=\left(T-T_{\mathrm{c}}\right) / T_{\mathrm{c}}$ is a relative divergence of the temperature from its critical value; $\mu$ is the critical exponent for thermal expansivity (can be used instead of critical exponent of the coexistence curve $\beta$ in the supercritical region). That is we deal with the critical exponents for the isodynamic quantities.

Applying (7) to (6), we get 


$$
\alpha^{\prime}=\gamma^{\prime}=\mu^{\prime} .
$$

For the first kind linear phase transitions it can be expected that the coexistence line continuously run into the lowered stability line (the line supercritical transitions) if the slope of the equilibrium line is the same as the slope of the continuous transition line (such result was obtained in [11] for the ferroelectric Lieb model). Then, in a small neighborhood of such a critical point, the relation (8) must be fulfilled along the equilibrium line, and as a result of the continuity of the passing of the equilibrium line into the supercritical transitions line, a symmetry between the critical exponents should be observed. Thus, along the lowered stability line the relation should hold:

$$
\alpha=\gamma=\mu .
$$

It is worth to be noted that this finding are true when the crical slope of the phase equilibrium curve is constant, but non-zero. When $K_{\mathrm{c}}=\Delta S / \Delta V=0$, we have $\gamma^{\prime} \geq \mu^{\prime}$, $\gamma^{\prime} \geq \alpha^{\prime}, \mu^{\prime} \geq \alpha^{\prime}$ and in supercritical region $\gamma \geq \mu, \gamma \geq \alpha, \mu \geq \alpha$. We have such a case for the Lieb model [11] and for the Curie-Weiss model when the line of phase coexistence is $H=0$ (Curie-Weiss) or $E=0$ (Lieb). Here $H, E$ are strengths of magnetic and electric fields, respectively.

If we introduce the critical exponents for the adiabatic quantities $(\partial T / \partial S)_{V}$, $(-\partial P / \partial V)_{s},(\partial T / \partial V)_{S}$ and denote them as $\alpha_{1}, \gamma_{1}, \mu_{1}$, we obtain the same relationships for the critical exponents as in the case of isodynamical quantities: $\alpha_{1}=\gamma_{1}=\mu_{1}$ for $K_{\mathrm{c}}=$ const $\neq\{0, \infty\}$ and $\gamma_{1} \geq \mu_{1}, \gamma_{1} \geq \alpha_{1}, \mu_{1} \geq \alpha_{1}$ for $K_{\mathrm{c}}=0$.

\section{Application to classical models}

The Curie-Weiss equation for ferromagnetism of electron gas [12] can be written as

$$
H=-\lambda M+\frac{N k T}{M_{0}} \operatorname{Arth}\left(\frac{M}{M_{0}}\right)
$$

where $M$ is a magnetization, $\lambda$ is Weiss coefficient, $M_{0}=N \mu_{\mathrm{B}}$ ( $\mu_{\mathrm{B}}$ is Bohr magneton), $k$ is Boltzmann constant. We can find all the set of thermodynamic characteristics:

$$
\begin{aligned}
& \left(\frac{\partial H}{\partial M}\right)_{T}=\frac{N k T}{M_{0}^{2}-M^{2}}-\lambda, \quad\left(\frac{\partial T}{\partial S}\right)_{M}=\frac{T}{C_{M}}=\text { const, } \\
& \left(\frac{\partial H}{\partial M}\right)_{S}=\frac{N k T}{M_{0}^{2}-M^{2}}-\lambda-\frac{N^{2} k^{2}}{M_{0}^{2}} \frac{T}{C_{M}} \operatorname{Arth}^{2}\left(\frac{M}{M_{0}}\right), \\
& \left(-\frac{\partial T}{\partial M}\right)_{H}=\left(\frac{N k T}{M_{0}^{2}-M^{2}}-\lambda\right) \frac{M_{0}}{N k} \operatorname{Arth}^{-1}\left(\frac{M}{M_{0}}\right),\left(\frac{\partial T}{\partial M}\right)_{S}=\frac{T}{C_{M}} \frac{N k}{M_{0}} \operatorname{Arth}\left(\frac{M}{M_{0}}\right), \\
& \left(\frac{\partial T}{\partial S}\right)_{H}=\left[\frac{C_{M}}{T}+\left(\frac{N k T}{M_{0}^{2}-M^{2}}-\lambda\right)^{-1} \frac{N^{2} k^{2}}{M_{0}^{2}} \operatorname{Arth}^{2}\left(\frac{M}{M_{0}}\right)\right]^{-1} .
\end{aligned}
$$

According to the thermodynamic classification of the types of critical behavior of thermodynamic systems, this correspond to the second type of critical behavior with $T / C_{M}=$ const, $T / C_{H}=$ const, $K_{\mathrm{c}}=0,(\partial H / \partial M)_{T}$ and $(\partial H / \partial M)_{S}$ becoming zero at the critical point. This type of critical behavior is fraught with the moderate energy 
flectuations and very high level of magnetic susceptibility fluctuations. The critical exponents of the model take the classical values $\alpha=\alpha^{\prime}=0, \beta=1 / 2, \gamma=\gamma^{\prime}=1$. And we can see that inequalities $\gamma^{\prime}>\alpha^{\prime}, \gamma>\alpha$ are fulfilled as the strict ones.

The thermal equation of state for the model is linear in temperature. So, according to the previous general consideration, the line of supercritical transition for the isodynamic magnetic coefficient of stability $(\partial H / \partial M)_{T}$ and for the deperminant of stability coincides and is determined as $\left(\partial^{2} H / \partial M^{2}\right)_{T}=0$. This gives us the solution $H=0$. Thus, for the Curie-Weiss model the supercritical transition line passes into the subcritical coexistense line (binodal, which is also the spinodal for this model) in a continuous manner. Such a conjugation at the critical point was proved to observed in the six-vertex Lieb model [11].

The van der Waals state equation [3] is $P=\frac{N k T}{V-b N}-\frac{a N^{2}}{V^{2}}$, where $b$ is an effective volume of molecule, $a$ is an interaction constant. The obtained thermodynamic quantities for the model are:

$$
\begin{aligned}
& \left(-\frac{\partial P}{\partial V}\right)_{T}=\frac{N k T}{(V-b N)^{2}}-\frac{2 a N^{2}}{V^{3}}, \quad\left(\frac{\partial T}{\partial S}\right)_{V}=\frac{T}{C_{V}}=\mathrm{const}, \\
& \left(\frac{\partial T}{\partial S}\right)_{P}=\left[\frac{C_{V}}{T}+\frac{N^{2} k^{2}}{(V-b N)^{2}}\left[\frac{N k T}{(V-b N)^{2}}-\frac{2 a N^{2}}{V^{3}}\right]^{-1}\right]^{-1},\left(\frac{\partial T}{\partial V}\right)_{S}=-\frac{N k T}{C_{V}(V-b N)}, \\
& \left(-\frac{\partial P}{\partial V}\right)_{S}=\frac{N k T}{(V-b N)^{2}}-\frac{2 a N^{2}}{V^{3}}+\frac{N^{2} k^{2} T}{C_{V}(V-b N)^{2}}, \\
& \left(\frac{\partial T}{\partial V}\right)_{P}=\frac{V-b N}{N k}\left[\frac{N k T}{(V-b N)^{2}}-\frac{2 a N^{2}}{V^{3}}\right] .
\end{aligned}
$$

At the critical point all adiabatic quantities are non-zero constant, and all isodynamic values approaches zero in the same manner. So, $\alpha_{1}=\gamma_{1}=\mu_{1}=1$.

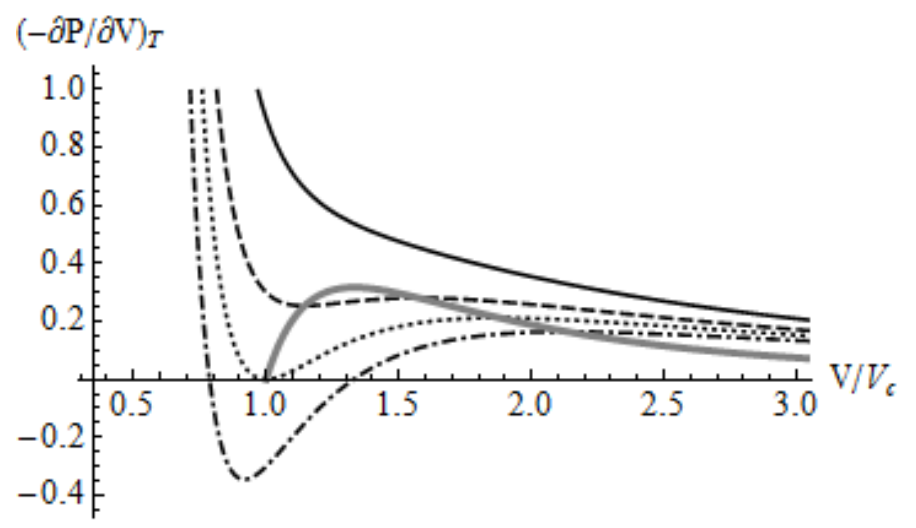

Fig. 1. The isotherms of relative mechanical stability coefficient $\left(-\cdot-\cdot--T / T_{\mathrm{c}}=0.95, \ldots \ldots . .-T / T_{\mathrm{c}}=1.0\right.$, $---T / T_{\mathrm{c}}=1.05$, black solid curve $-T / T_{\mathrm{c}}=1.15$ ) and quasispinodal (gray bold curve) for the van der Waals model.

This correspond to the first type of critical behavior [7] with small fluctuations of energy and compressibility. 
The equation of the supercritical transition according to our consideration is $\left(-\partial^{2} P / \partial V^{2}\right)_{T}$. And this gives us the following expression: $T=3 a N(V-b N)^{3} / k V^{4}$. It is shown as a gray line in Fig. 1, where results are plotted in relative values $P / P_{\mathrm{c}}, V / V_{\mathrm{c}}$, $T / T_{\mathrm{c}}$, and $P_{\mathrm{c}}, V_{\mathrm{c}}, T_{\mathrm{c}}$ are critical parameters for the van der Waals model:

$$
P_{\mathrm{c}}=a /\left(27 b^{2}\right), V_{\mathrm{c}}=3 b N, T_{\mathrm{c}}=8 a /(27 b k) .
$$

\section{Conclusions}

In this paper we consider the breakdown of thermodynamic stability of a simple one-component system in the supercritical region. We examine the methods for obtaining the lowered stability curve equation and ascertain when they are interchangeable. Thus, it is possible when we consider a system with a thermal state equation linear in temperature. Then the curve of the continuous transitions for the coefficients of stability and that for the determinant of stability coincides. We obtain the relationship between the critical exponents of the main thermodynamic characteristics and establish the condition when the supercritical transitions line continuously conjugates to binodal at the critical point. The obtained results were applied to the classical models of liquid and magnetic systems: the Curie-Weiss and the van der Waals ones.

\section{References}

1. Semenchenko, V.K. Termodinamicheskaya ustojchivost i fazovye perehody v kristallah / V.K. Semenchenko // Kristallografiya. - 1964. - Vol. 9, No. 5. - P. 611 - 621.

2. Galdina, A.N. Supercritical behavior of thermodynamic systems / A.N. Galdina // Journal of Physics and Electronics. - 2019. - Vol. 27, No. 1. - P. 19 - 26.

3. Bulavin, L.A. Termodynamika rozplaviv / L.A. Bulavin, O.O. Klyuchnykov, Yu.O. Plevachuk et al. - Chornobyl: Instytut problem bezpeky AES, 2014. - 388 p.

4. Poberezhnyuk, R.V. Quantum van der Waals and Walecka models of nuclear matter / R.V. Poberezhnyuk, V. Vovchenko, D.V. Anchishkin, and M.I. Gorenstein // International Journal of Modern Physics E. - 2017. - Vol. 26, No. 10. - 1750061 (14 p.).

5. Fortov, V.Ye. Uravneniya sostoyaniya veshchestva: ot ideal'noho gaza do kvarkgluonnoj plazmy / V.Ye. Fortov. - Moskva: Fizmatlit, 2012. - 492 p.

6. Fortov, V.Ye. Fizika vysokih plotnostej energii / V.Ye. Fortov. - Moskva: Fizmatlit, 2013. - $712 \mathrm{p}$.

7. Soldatova, E.D. Variety of critical state nature manifestations / E.D. Soldatova // Condensed Matter Physics. - 1999. - Vol. 2, No. 4 (20). - P. 603 - 616.

8. Soldatova, E.D. Thermodynamic stability of equilibrium system and critical state / E.D. Soldatova // Journal of Molecular Liquids. - June 2005. - Vol. 120, Issue 1-3. - P. $47-50$.

9. Soldatova, E.D. Stability conditions for the basic thermodynamic potentials and the substantiation of the phase diagrams / E.D. Soldatova // Journal of Molecular Liquids. - 2006. - Vol. 127, Issue 1-3. - P. 99 - 101.

10. Stanley, E.H. Introduction to phase transition and critical phenomena / E.H. Stanley. - Oxford University Press, 1987. - 308 p.

11. Galdina, A.N. Rivnyannya linii znyzhenoji stijkosti dlya modeli Liba / A.N. Galdina // Visnyk Dnipropetrovskogo universitetu. Serija Fizika. Radioelektronika. - 2007. - Vol. 14, Issue 12/1. - P. 68 - 70.

12. Rumer, Yu.B. Termodinamika, statisticheskaya fizika i kinetika / Yu.B. Rumer, M.Sh. Ryvkin // Novosibirsk: Izd-vo Novosibirskogo Universiteta, 2000. - 608 p. 